\title{
Stimulatory Effects of Flusilazole on Virulence of Sclerotinia sclerotiorum
}

\author{
Xiaoming Lu, Ran Zhang, Menglong Cong, Jianhong Li, and Fuxing Zhu, ${ }^{\dagger}$ College of Plant Science and Technology, Huazhong Agri- \\ cultural University, Wuhan, 430070, China
}

\begin{abstract}
Flusilazole, a member of the demethylation inhibitor fungicides, is highly efficacious for control of Sclerotinia sclerotiorum. To achieve judicious applications of flusilazole, its hormetic effects on virulence of S. sclerotiorum were investigated. Flusilazole sprayed at concentrations from 0.02 to $0.5 \mu \mathrm{g} / \mathrm{ml}$ caused statistically significant $(P<0.05)$ stimulatory effects on virulence of $S$. sclerotiorum to potted rapeseed plants, and the maximum stimulation magnitudes were 11.0 and $10.7 \%$ for isolates GS-7 and HN-24, respectively. Studies on the time course of the infection process showed that a stimulatory effect on virulence could be discerned at $18 \mathrm{~h}$ postinoculation, indicating a direct stimulation mechanism rather than an overcompensation for initial inhibitions. In order to determine whether the stimulations were caused mainly by effects of flusilazole on $S$. sclerotiorum or on rapeseed plants, mycelia grown on flusilazoleamended potato dextrose agar (PDA) media were inoculated on leaves

of rapeseed plants without spraying the fungicide. Mycelium radial growth on PDA supplemented with flusilazole at concentrations from 0.005 to $0.16 \mu \mathrm{g} / \mathrm{ml}$ was inhibited by 10.11 to $48.7 \%$ for isolate GS-7 and by 4.1 to $24.9 \%$ for isolate $\mathrm{HN}-24$. Observations with a scanning electron microscope showed that flusilazole in PDA at 0.04 and $0.08 \mu \mathrm{g} / \mathrm{ml}$ caused slightly deformed mycelia and twisted mycelial tips. Nevertheless, after inoculating on leaves of potted rapeseed plants, virulence of the inhibited mycelia was statistically significantly $(P<0.05)$ greater than that of the nontreated control, and the maximum stimulation magnitudes were 16.2 and $19.8 \%$ for isolates GS-7 and HN-24, respectively. Studies on a physiological mechanism for virulence stimulations showed that tolerance to hydrogen peroxide did not increase significantly for mycelia grown on flusilazole-amended PDA, thus excluding the possibility of tolerance to reactive oxygen species as a potential mechanism for virulence stimulations.
\end{abstract}

The ascomycete fungus Sclerotinia sclerotiorum (Lib.) de Bary is a necrotrophic plant pathogen notorious for its broad host range of more than 400 plant species in 75 families, including many economically important crops and vegetables such as rapeseed, soybean, sunflower, and tomato (Boland and Hall 1994; Bolton et al. 2006). Crop diseases caused by $S$. sclerotiorum have always been difficult to control due to limited genetic resources for $S$. sclerotiorum resistance in major crops and long survival ability of sclerotia in the soil (Adams and Ayres 1979). S. sclerotiorum may cause heavy yield losses in many crops and it has been reported that rapeseed production losses due to Sclerotinia stem rot range from 10 to $80 \%$ annually in China (Wang et al. 2014). In the United States, annual economic losses caused by $S$. sclerotiorum in five crops (i.e., soybean, sunflower, dry editable bean, canola, and pulse) are about $\$ 482$ million (USDA 2012).

Among the integrated disease management tactics, fungicides remain the mainstay for the control of S. sclerotiorum. Sterol demethylation inhibitor (DMI) fungicides have been widely used in agricultural and medical settings against a wide variety of pathogenic fungi in crops, animals, and humans (Becher and Wirsel 2012). The DMI fungicide flusilazole is a broad-spectrum fungicide with highly preventative and curative efficacies against $S$. sclerotiorum (Lu et al. 2015). At present, flusilazole has not been registered in China to control diseases caused by $S$. sclerotiorum (http://www.chinapesticide. gov.cn/hysj/index.jhtml). Due to evaporation, drift, or uneven penetration into the crop canopy, fungicide spraying in the field will inevitably lead to exposure to sublethal doses of fungicides, at least for a portion of pathogens or isolates (Flores and Garzón 2013). Sublethal doses of fungicides may increase rather than reduce mycelial radial growth on artificial media and virulence on host plants. Stimulatory effects have been reported for thiabendazole on germination of spores of Penicillium expansum (Baraldi et al. 2003), propamocarb and cyazofamid on mycelial growth of Pythium aphanidermatum (Flores and Garzón 2013), metalaxyl on mycelial growth of

${ }^{\dagger}$ Corresponding author: F. Zhu; E-mail: zhufuxing@mail.hzau.edu.cn

Accepted for publication 14 September 2017.

@ 2018 The American Phytopathological Society
Phytophthora infestans (Zhang et al. 1997), and mefenoxam on mycelial growth and virulence of Pythium aphanidermatum (Garzón et al. 2011; Moorman and Kim 2004). With respect to S. sclerotiorum, stimulatory effects on virulence have been reported for dimethachlone, carbendazim, and trifloxystrobin (Di et al. 2015, 2016a; Zhou et al. 2014). Studies with detached rapeseed leaves showed that spraying dimethachlone at $300 \mathrm{mg} /$ liter increased virulence of an isolate of S. sclerotiorum resistant in the field (Firoz et al. 2016). Stimulatory effects of low doses of fungicides on virulence of fungal plant pathogens have much relevance to management of fungal diseases. The toxic activity of flusilazole on $S$. sclerotiorum has been reported (Lu et al. 2015). The objectives of the present study were to (i) assess stimulatory effects of sublethal doses of flusilazole on virulence of $S$. sclerotiorum to rapeseed plants, (ii) determine the time course of the stimulations on virulence of $S$. sclerotiorum, and (iii) elucidate possible physiological mechanisms for the stimulatory effects of flusilazole.

\section{Materials and Methods}

Collection and preservation of isolates of $S$. sclerotiorum. Two isolates of S. sclerotiorum, HN-24 and GS-7, were collected in 2012 from rapeseed fields in Hunan and Gansu Provinces, respectively. The collected mature sclerotia were stored at $4{ }^{\circ} \mathrm{C}$ in a refrigerator. Both isolates were sensitive to flusilazole and the effective concentrations at which mycelial growth rate on potato dextrose agar (PDA) was inhibited by $50 \%$ ( $\mathrm{EC}_{50}$ values) for isolates $\mathrm{HN}-24$ and GS-7 were 0.085 and $0.104 \mu \mathrm{g} / \mathrm{ml}$, respectively (Lu et al. 2015).

Fungicide. A technical-grade formulation of flusilazole (96.4\% active ingredient; Hubei Kang Bao Tai Fine-Chemical Co. Ltd.) was stored at $4^{\circ} \mathrm{C}$ in a refrigerator and a stock solution at $1,000 \mu \mathrm{g} / \mathrm{ml}$ was prepared by dissolving the fungicide in pure acetone.

Stimulatory effects of spraying low doses of flusilazole on virulence of $S$. sclerotiorum to potted rapeseed plants. The procedure for assessing stimulatory effects of spraying flusilazole was according to Di et al. (2016a). Stock solution of flusilazole was serially diluted with $0.1 \%$ Triton-100 in water to concentrations of $8,4,2,0.5$, $0.2,0.05,0.02,0.002$, and $0.0002 \mu \mathrm{g} / \mathrm{ml}$. Flusilazole solutions were sprayed until runoff on 4-week-old rapeseed plants growing in the greenhouse. Rapeseed plants sprayed with $0.1 \%$ Triton- 100 in water were used as the nontreated control. After air drying for $1 \mathrm{~h}$, two leaves per plant were inoculated on the adaxial surface with inverted mycelial plugs (5 $\mathrm{mm}$ in diameter), with one mycelial plug for each 
leaf. The inoculated plants were incubated at $23^{\circ} \mathrm{C}$ with photoperiod of 16 and $8 \mathrm{~h}$ (light and darkness, respectively) and relative humidity was maintained at about $85 \%$. After $48 \mathrm{~h}$ of incubation, lesion diameters were measured twice in two perpendicular directions. All experiments in the study were performed in triplicate and repeated at least twice.

Stimulatory effects of growing on flusilazole-amended PDA on virulence to potted rapeseed plants. The procedure was according to Zhou et al. (2014). Inverted mycelial plugs were cut from 2-day-old cultures growing on PDA supplemented with flusilazole at final concentrations of $0.0025,0.005,0.04,0.1,0.16,0.25$, or $0.32 \mu \mathrm{g} / \mathrm{ml}$, and were inoculated, with the mycelium side down, on the adaxial leaf surface of 4-week-old rapeseed plants. Mycelial plugs from a colony on PDA supplemented with acetone at a final concentration of $0.5 \%$ ( $\mathrm{vol} / \mathrm{vol})$ were used as the nontreated control. The inoculated plants were incubated and lesion diameters were measured as described above.

Time course of stimulation on virulence of $S$. sclerotiorum. The procedure for assessing the time course of virulence stimulation was according to Di et al. (2016a,b). Rapeseed plants were grown at $23^{\circ} \mathrm{C}$ in a greenhouse for about 4 weeks, and three leaves of 8 to $11 \mathrm{~cm}$ in diameter were detached from each plant. The detached leaves were rinsed with sterile water, air dried for $1 \mathrm{~h}$ in the laboratory, and sprayed with flusilazole dilutions at 0.002 and $0.02 \mu \mathrm{g} / \mathrm{ml}$ by a hand-held sprayer ( $800 \mathrm{ml}$ in volume; Xinmeir Co. Ltd.). Leaves sprayed with acetone in $0.1 \%$ Triton- 100 at a final concentration of $0.5 \%$ ( vol/vol) were employed as the nontreated control. After being air dried for about $1 \mathrm{~h}$, leaves were transferred into Petri dishes $(15 \mathrm{~cm}$ in diameter) that were embedded with a piece of wetted filter paper to maintain high humidity. The treated leaves were then
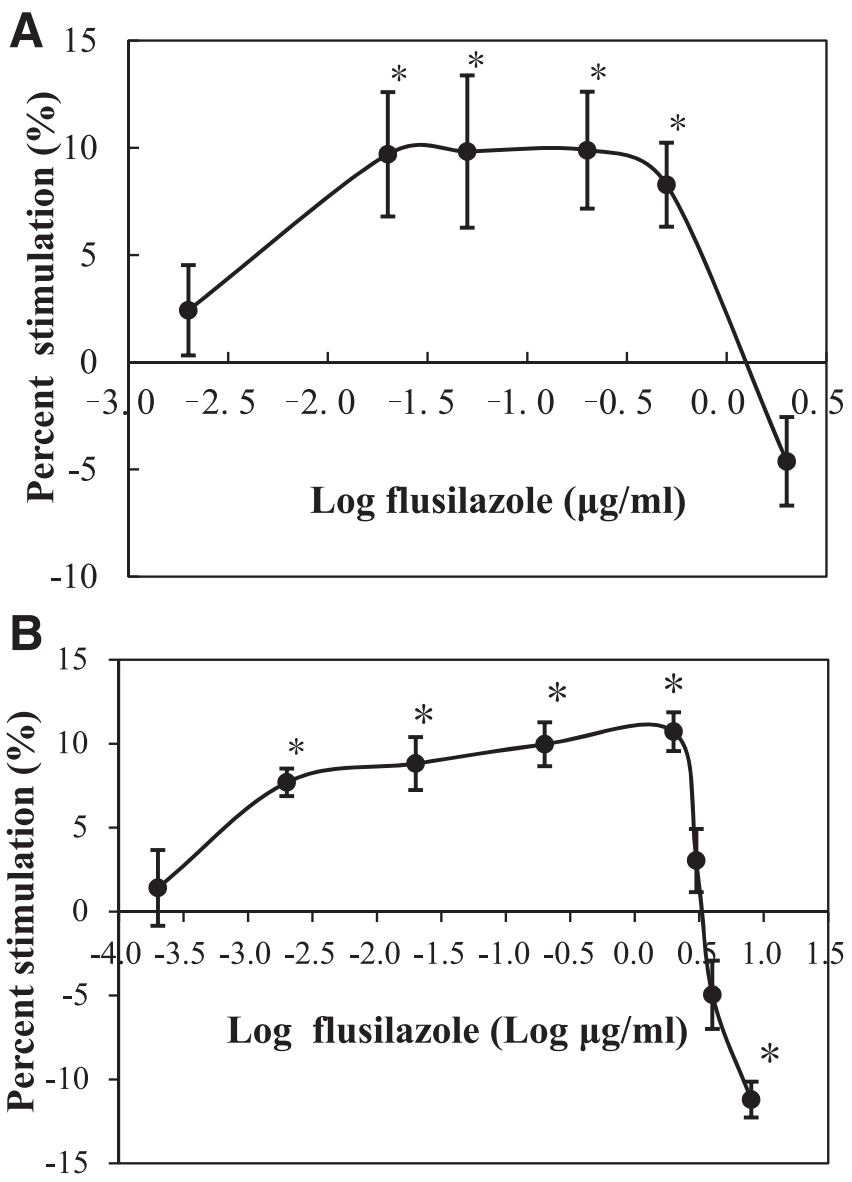

Fig. 1. Stimulatory effects of spraying low doses of flusilazole on virulence of Sclerotinia sclerotiorum A, isolate GS-7 and B, isolate $\mathrm{HN}-24$ on potted rapeseed plants. Error bar denotes standard error of the mean. Asterisk denotes significant difference from the control at $\alpha=0.05$. Data are mean values of 12 replicates. inoculated on the adaxial surface with inverted mycelial plugs, as described above. Petri dishes were lidded and the inoculated leaves were incubated at $23^{\circ} \mathrm{C}$, with relative humidity maintained at about $85 \%$. Disease symptoms were photographed with a digital camera (Canon Powershot G12) at 12, 18, 24, 36, and $48 \mathrm{~h}$ postinoculation (HPI).

Effects of low doses of flusilazole on tolerance capacity of S. sclerotiorum to hydrogen peroxide. Two-day-old mycelial colonies grown on PDA were subcultured on flusilazole-supplemented PDA. The final concentrations of flusilazole in PDA were 0.02 , $0.04,0.08,0.16$, and $0.32 \mu \mathrm{g} / \mathrm{ml}$. Mycelia subcultured on PDA supplemented with $0.5 \%(\mathrm{vol} / \mathrm{vol})$ acetone were used as the fungicide-free control. After the PDA plates had been incubated at $23^{\circ} \mathrm{C}$ for $48 \mathrm{~h}$, mycelial plugs cut from actively growing margins of colonies were transferred to PDA media supplemented with hydrogen peroxide $\left(\mathrm{H}_{2} \mathrm{O}_{2}\right)$ at final concentrations of 0 (without $\mathrm{H}_{2} \mathrm{O}_{2}$ ), 200,400, or $800 \mu \mathrm{g} / \mathrm{ml}$. After growing at $23^{\circ} \mathrm{C}$ for $48 \mathrm{~h}$, mycelial growth rates were determined by measuring each colony diameter twice at right angles.

Electron microscopic observations on effects of flusilazole on mycelial morphology of $\boldsymbol{S}$. sclerotiorum. The procedure was according to Lu et al. (2015). Mycelia were grown for $48 \mathrm{~h}$ on flusilazole-amended PDA media overlaid with a piece of cellophane. The final concentrations of flusilazole in PDA were 0.04 and $0.08 \mu \mathrm{g} / \mathrm{ml}$. Mycelia grown on cellophane-overlaid PDA media supplemented with acetone at $0.5 \%$ ( $\mathrm{vol} / \mathrm{vol})$ were employed as the fungicide-free control. Samples were collected from 2-day-old colonies by cutting growing margins into small pieces of 3 by $3 \mathrm{~mm}$ with a scalpel. The samples were fixed at $4{ }^{\circ} \mathrm{C}$ for $24 \mathrm{~h}$ in $2.5 \%(\mathrm{wt} / \mathrm{vol})$ glutaraldehyde solution in sodium phosphate buffer $(0.05 \mathrm{M}, \mathrm{pH}$ 7.0). After being rinsed three times (10 min each time) in $0.05 \mathrm{M}$ sodium phosphate buffer, the samples were dehydrated in a graded ethanol series. After critical point drying and gold coating in a sputter coater (JFC-1600; NTC), the leaf specimens were examined with a scanning electron microscope (SEM; JSM-6390/ LV; NTC).

Data processing and analysis. Percent stimulations of low doses of flusilazole on virulence were calculated by the formula: percent stimulation $(\%)=[($ lesion diameter of the treated - lesion diameter of the control)/lesion diameter of the control] $\times 100 \%$. Arcsine square-root transformation was performed on the percent stimulation data before statistical analysis. Replicate data from different repetitions of the same experiment were pooled together because differences among repetitions were not significant. The multiple comparison procedure of Dunnett's test $(\alpha=0.05)$ in SPSS (Statistical Product and Service Solutions, ver. 21.0; SPSS Inc.) was employed to assess statistical significance of percent stimulation of each treatment compared with the nontreated control. Figures were constructed with Microsoft Excel software (ver. 2010; Microsoft Corporation).

\section{Results}

Stimulatory effects of spraying low doses of flusilazole on virulence of $\boldsymbol{S}$. sclerotiorum. Spraying flusilazole at 0.02 to $0.5 \mu \mathrm{g} / \mathrm{ml}$ onto potted rapeseed plants elicited statistically significant $(P<0.05)$ stimulations of virulence of isolate GS-7 (Fig. 1A). Compared with the fungicide-free control, the maximum stimulation amplitude was $11 \%$, at which the concentration of flusilazole was $0.2 \mu \mathrm{g} / \mathrm{ml}$. For isolate $\mathrm{HN}-24$, the stimulatory range of flusilazole was from 0.002 to $2 \mu \mathrm{g} / \mathrm{ml}$ and the stimulation amplitude was from 7.7 to $10.7 \%$ (Fig. 1B). For both isolates, the stimulatory curves did not follow the shape of a typical parabola.

Stimulatory effects of growing on PDA supplemented with flusilazole on virulence to potted rapeseed plants. Flusilazole in PDA at concentrations from 0.005 to $0.16 \mu \mathrm{g} / \mathrm{ml}$ inhibited mycelial growth rates by 10.1 to $48.4 \%$ for isolate GS-7 and by 4.1 to $24.9 \%$ for isolate HN-24. However, after these inhibited mycelia were inoculated on potted rapeseed plants, virulence (i.e., lesion diameter) increased statistically significantly $(P<0.05)$, compared with the nontreated control (Fig. 2). The maximum percent stimulations were 16.2 and $19.5 \%$ for isolates GS-7 and $\mathrm{HN}-24$, respectively. Flusilazole in 
PDA media at $0.32 \mu \mathrm{g} / \mathrm{ml}$ showed inhibitory effects on virulence for both isolates.

Effects of flusilazole on tolerance capacity of $S$. sclerotiorum to $\mathrm{H}_{2} \mathrm{O}_{2}$. Compared with mycelial growth rates on PDA without $\mathrm{H}_{2} \mathrm{O}_{2}$, growth rates on PDA amended with $\mathrm{H}_{2} \mathrm{O}_{2}$ at 200, 400, or $800 \mu \mathrm{g} / \mathrm{ml}$ were strongly inhibited (Fig. 3). However, no statistically significant difference $(P>0.05)$ in mycelial growth rate was detected among different concentrations of flusilazole, indicating that flusilazole had no obvious effect on the tolerance capacity of S. sclerotiorum to $\mathrm{H}_{2} \mathrm{O}_{2}$.

Time course of virulence stimulation. At $12 \mathrm{HPI}$, neither the flusilazole treatment nor the nontreated control group showed any sign of necrotic lesions (Fig. 4). At $18 \mathrm{HPI}$, differences in the development of disease symptoms could be discerned with the naked eye. Early signs of infections were visible only on leaves sprayed with flusilazole at $0.002 \mu \mathrm{g} / \mathrm{ml}$, whereas no symptoms of infections could be discerned with the naked eye for the nontreated control, indicating a direct stimulation mechanism. From 24 to 36 HPI, disease symptoms developed obviously faster on leaves sprayed with flusilazole at 0.002 and $0.02 \mu \mathrm{g} / \mathrm{ml}$ than on the nontreated control leaves.

Effects of flusilazole on mycelial morphology of S. sclerotiorum. Microscopic observations with an SEM demonstrated that hyphae without flusilazole treatment seemed natural (Fig. 5). The tips of hyphae grown on PDA supplemented with flusilazole at $0.04 \mu \mathrm{g} / \mathrm{ml}$ were contorted, twisted, and more branched. The hyphae grown on PDA supplemented with flusilazole at $0.08 \mu \mathrm{g} / \mathrm{ml}$ shrank and shriveled. It
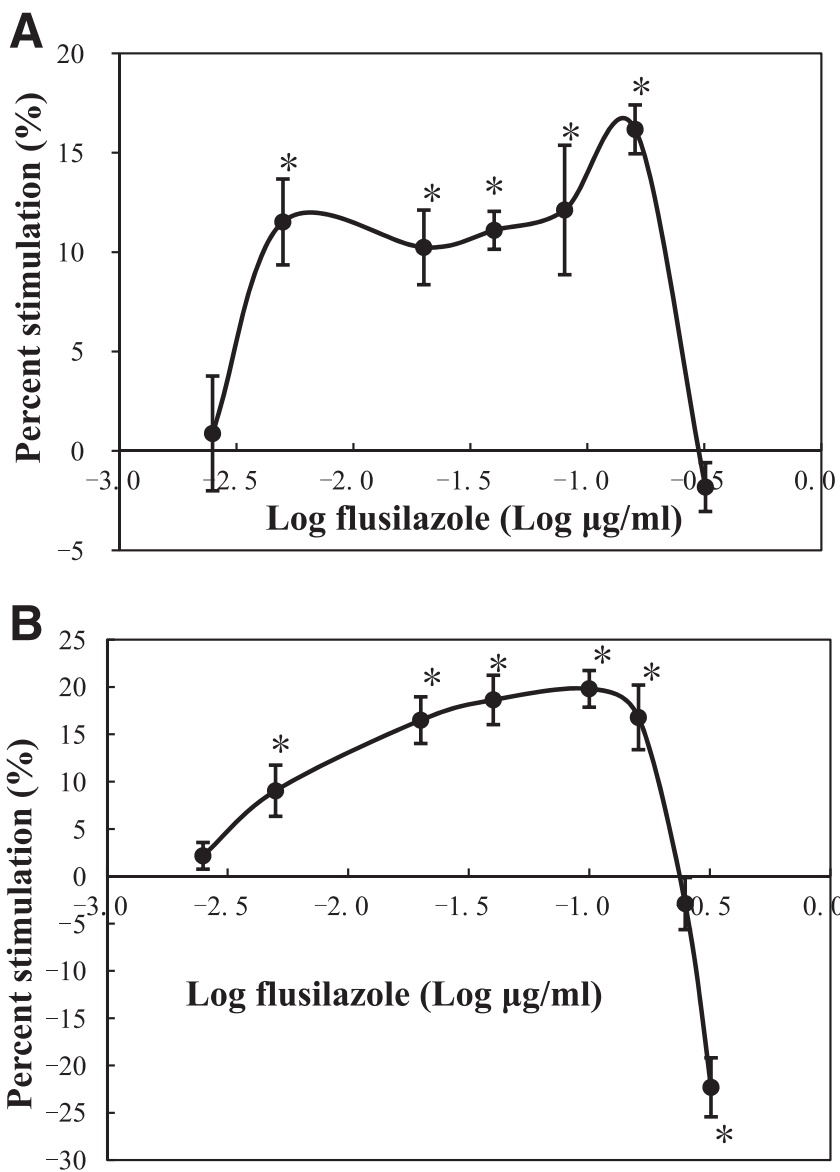

Fig. 2. Stimulatory effect of growing on flusilazole-amended potato dextrose agar (PDA) on virulence of Sclerotinia sclerotiorum isolates A, GS-7 and B, HN-24 on potted rapeseed plants. Error bar denotes standard error of the mean. Asterisk denotes significant differences from the control at $\alpha=0.05$. Data are mean values of 12 replicates. For isolate GS-7, mycelial growth inhibitions on PDA supplemented with flusilazole at $0.0025,0.005,0.02,0.04,0.08,0.16$, and $0.32 \mu \mathrm{g} / \mathrm{ml}$ were $3.9,10.1$, 15.7, 25.2, 39.6, 48.4, and 59.7\% respectively. For isolate $\mathrm{HN}-24$, mycelial growth inhibitions on PDA supplemented with flusilazole at $0.0025,0.005,0.02,0.04,0.1$, 0.16 , and 0.32 were $2.2,4.1,3.5,9.5,14.4,24.9$, and $41.0 \%$, respectively. was apparent that flusilazole at 0.04 and $0.08 \mu \mathrm{g} / \mathrm{ml}$ inhibited the normal growth of mycelia and substantially affected mycelial morphology of S. sclerotiorum.

\section{Discussion}

Our previous studies showed that flusilazole sprayed at $90 \mu \mathrm{g} / \mathrm{ml}$ provided preventive efficacy of $85 \%$ and curative efficacy of $59 \%$ in potted experiments (Lu et al. 2015). The present study demonstrated that spraying flusilazole at doses lower than $1 \mu \mathrm{g} / \mathrm{ml}$ had significant stimulatory effect on virulence of $S$. sclerotiorum to rapeseed plants whereas, at concentrations higher than $4 \mu \mathrm{g} / \mathrm{ml}$, flusilazole showed inhibitory effects on virulence. The inverted U-shaped, biphasic responses of $S$. sclerotiorum to flusilazole conformed to the biological concept of hormesis. In toxicology, hormesis represents a kind of dose-response relationship characterized by low-dose stimulation, which is usually beneficial to the organism, and high-dose inhibition (Calabrese 2015a). As a generalizable phenomenon, studies and published papers on hormesis have dramatically increased in recent years. Quantitative characteristics of hormesis such as stimulation range for chemical or physical stresses and stimulation amplitude have been experimentally observed and thoroughly reviewed, especially by Calabrese $(2013,2015 a, b, 2016 a, b)$. Low-dose stimulations of mycelial growth and virulence of $S$. sclerotiorum have been reported for the fungicides dimethachlone, carbendazim, and trifloxystrobin (Di et al. 2015, 2016a; Zhou et al. 2014). The hormetic effects of trifloxystrobin were reported in sensitive isolates of S. sclerotiorum (Di et al. 2016a), whereas the stimulatory effects of carbendazim were reported in carbendazim-resistant isolates of S. sclerotiorum (Di et al. 2015). With respect to dimethachlone, the hormetic effects were observed in both dimethachlone-sensitive and -resistant isolates (Zhou et al. 2014). In the present study, stimulatory effects of flusilazole were assayed in sensitive isolates. For both sensitive and resistant isolates of $S$. sclerotiorum, stimulatory effects of fungicides were observed in a dose range immediately below the inhibitory dose range. This was an important feature of the concept of hormesis. In the study on dimethachlone hormesis, we reported that all three dimethachlone-resistant isolates and $24 \%$ of sensitive isolates displayed stimulatory responses to their respective sublethal doses of dimethachlone (Zhou et al. 2014). Our subsequent studies indicated that all isolates might display hormetic effects induced by low doses of a fungicide, as long as appropriate sublethal doses and adequate replicates are employed. Several replicates, as commonly used in ordinary experiments, may not have enough power to detect stimulatory effects, especially for sensitive wild-type

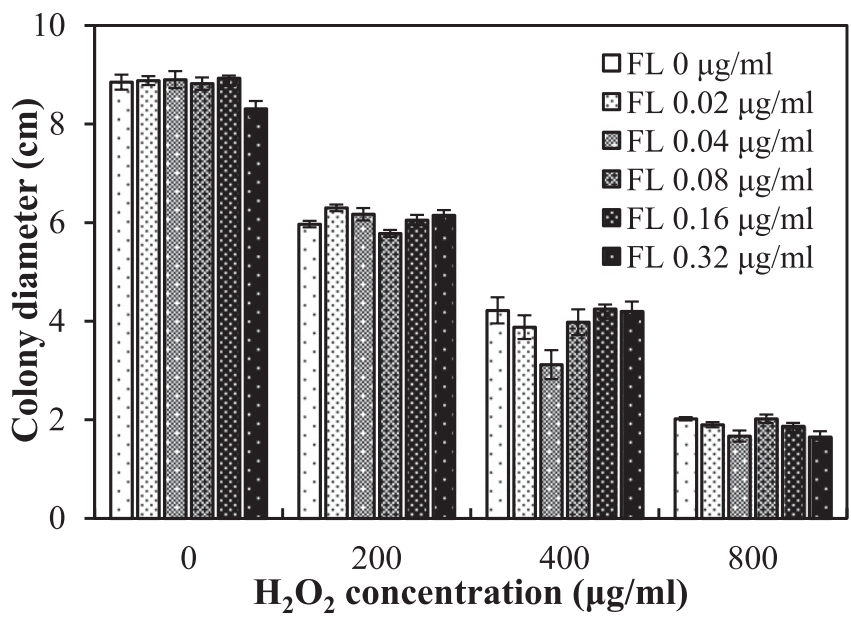

Fig. 3. Effects of growing on flusilazole-amended potato dextrose agar (PDA) on sensitivity of Sclerotinia sclerotiorum isolate $\mathrm{HN}-24$ to $\mathrm{H}_{2} \mathrm{O}_{2}$. $\mathrm{FL}$ denotes flusilazole. Error bar denotes standard error of the mean. Each column represents the mean value of six replicates. Mycelial growth inhibitions on PDA supplemented with flusilazole at $0.02,0.04,0.08,0.16$, and $0.32 \mu \mathrm{g} / \mathrm{ml}$ (as the pretreatment) were 5.1, 16.4, 23.9, 32.0, and $47.4 \%$, respectively. 
isolates, because of a mild stimulatory amplitude, as shown in the present study, where the maximum stimulations were only a little greater than $10 \%$. Thus, for studies on hormetic effects, a rigorous experiment design is needed (Flores and Garzón 2013). Another characteristic of hormetic dose-response is high fluctuations in stimulatory responses. In preliminary experiments of the present study, we found that stimulatory virulence and mycelial growth responses were not stable (data not shown). The relatively large error bars in Figures 1 and 2 also reflected the instability of stimulatory responses of $S$. sclerotiorum to flusilazole. The feature of high fluctuations in fungicide stimulations has also been reported by Garzón et al. (2011), whose studies showed that, although stimulations were observed in mycelial growth of $P$. aphanidermatum on corn meal agar supplemented with sublethal doses of mefenoxam in all repetitions, no statistically significant differences were found, mainly due to unstable responses. Whether the stimulation is stable or not, and whether the stimulation magnitude is great or small, our experience with studies on stimulatory effects of several fungicides indicates that fungicide hormesis should be a general and common phenomenon, at least in the ascomycete fungus $S$. sclerotiorum. The hormesis database established by Calabrese and Blain (2011) that contained approximately 9,000 dose responses indicated that hormesis is a generalizable, fundamental concept in biology, and has been reported in a wide range of biological models across a large variety of chemical classes.

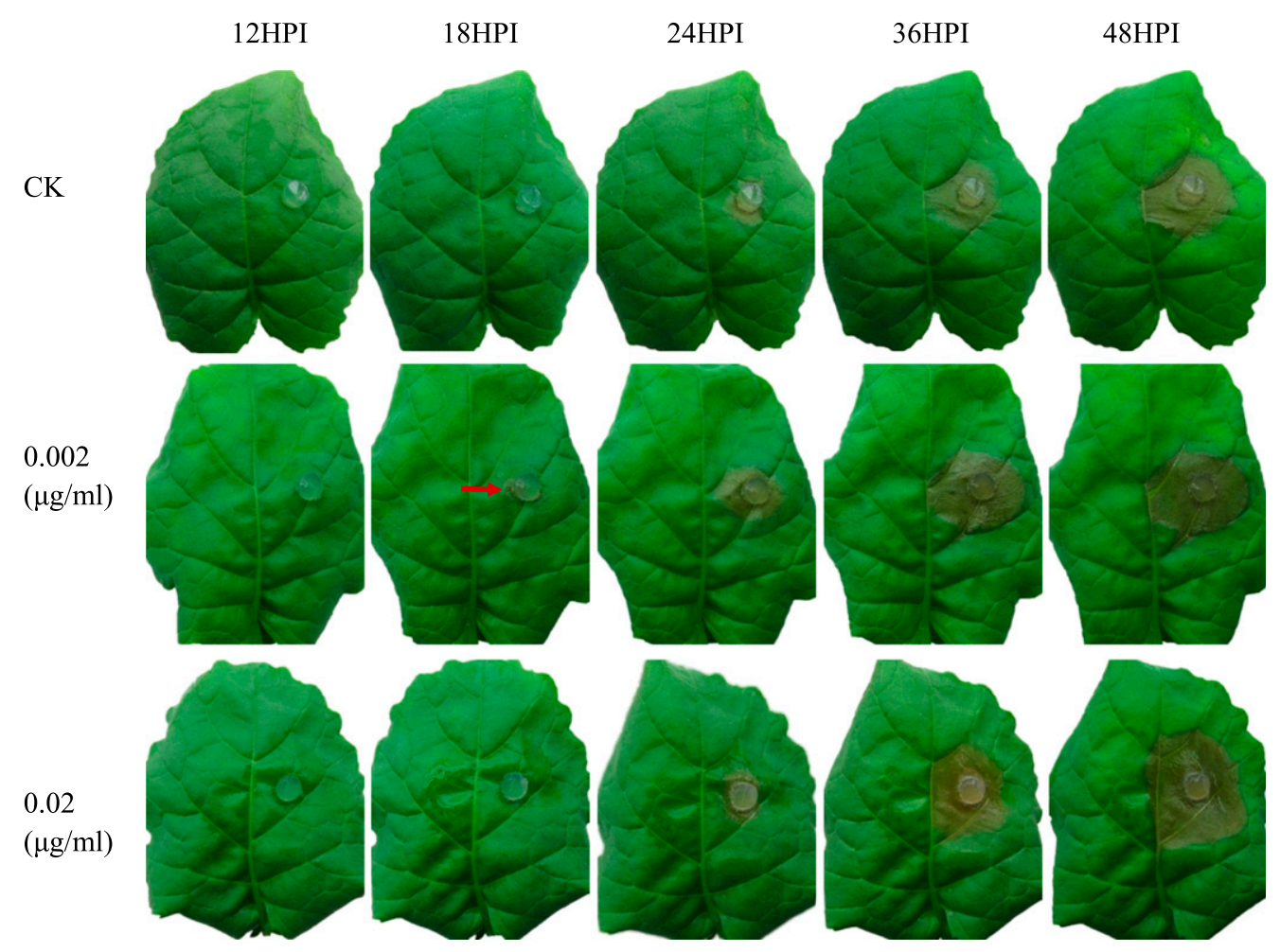

Fig. 4. Temporal profile of virulence stimulation of Sclerotinia sclerotiorum isolate GS-7 by low doses of flusilazole. Rapeseed leaves treated with $0.1 \%$ Triton-100 in water in the first row were used as the nontreated control (CK). HPI = hours postinoculation.
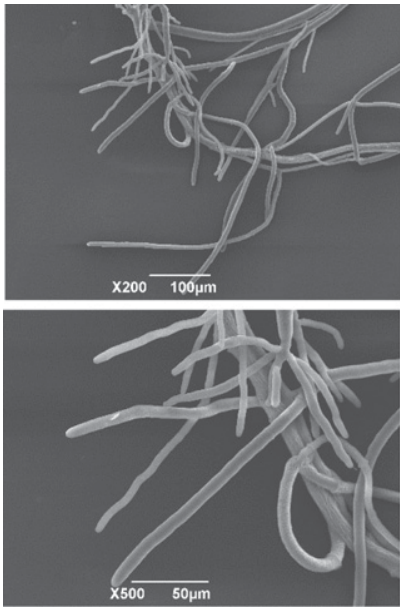

CK
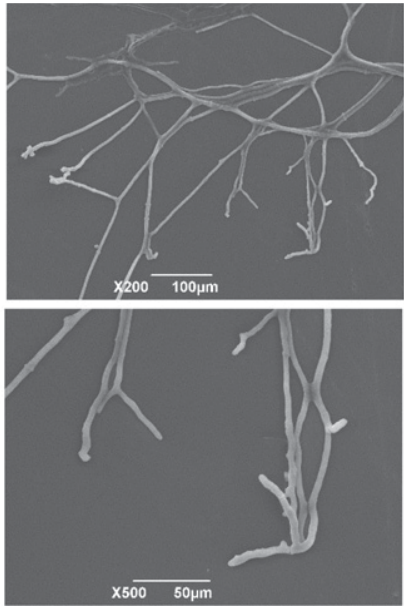

$0.04 \mu \mathrm{g} / \mathrm{ml}$

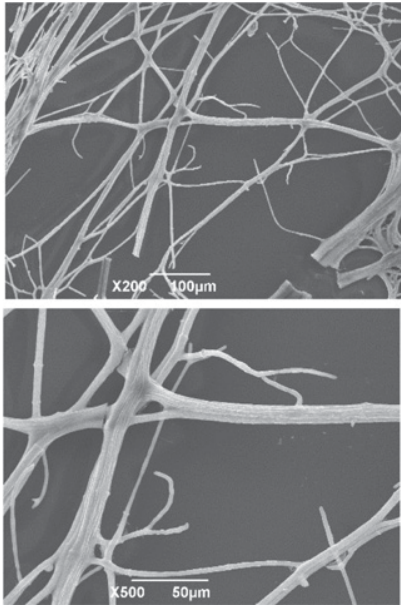

$0.08 \mu \mathrm{g} / \mathrm{ml}$

Fig. 5. Scanning electron micrographs showing effects of flusilazole on hyphal morphology of Sclerotinia sclerotiorum. In the first column, mycelia grown on potato dextrose agar amended with acetone at $0.5 \%$ (vol/vol) were used as the nontreated control (CK). 
With respect to hormetic mechanisms, hormetic dose-responses may be caused by direct stimulation or by an overcompensation to a disruption of homeostasis (Calabrese 2001). Numerous examples of direct stimulations and their descriptive and quantitative features have been reviewed and evaluated (Calabrese 2001). Our previous studies on the temporal profile of fungicide hormesis demonstrated that stimulatory virulence responses of $S$. sclerotiorum to low doses of carbendazim and trifloxystrobin were mediated by a direct stimulation mechanism (Di et al. 2016a,b). The present study also showed that virulence stimulations of flusilazole on S. sclerotiorum involved a direct stimulation mechanism. Fungal infection of plants is a complex process and plant physiological status may substantially affect progresses of fungal infections. Stimulations of low doses of flusilazole on virulence of $S$. sclerotiorum may be mainly mediated by effects of flusilazole on plant or on fungus. However, mycelia grown on flusilazole-amended PDA also exhibited enhanced virulence to oilseed plants without spraying fungicide, thus indicating that virulence stimulation was induced mainly through effects of the fungicide on mycelia of $S$. sclerotiorum. In the early contact and initial infection by a fungus, the plant will mount a common resistance response called oxidative burst, a rapid production and release of huge amounts of reactive oxygen species, including superoxide radical, hydroxyl radical, and $\mathrm{H}_{2} \mathrm{O}_{2}$ (Williams et al. 2011; Wojtaszek 1997). A high tolerance ability of the fungus is conductive to virulence. However, the present study demonstrated that growing on flusilazole-supplemented PDA did not increase the tolerance capacity of $\mathrm{S}$. sclerotiorum to $\mathrm{H}_{2} \mathrm{O}_{2}$, thus ruling out the possibility of tolerance to plant oxidative burst as a physiological mechanism for flusilazole stimulations. Similar results have been reported for the stimulatory effect of carbendazim on virulence of $S$. sclerotiorum (Di et al. 2015). Nevertheless, mycelia of S. sclerotiorum grown on PDA supplemented with dimethachlone exhibited higher tolerance capacity to $\mathrm{H}_{2} \mathrm{O}_{2}$ than the nontreated control (Zhou et al. 2014). These results demonstrated that effects on tolerance capacity of fungi to $\mathrm{H}_{2} \mathrm{O}_{2}$ varied among different classes of fungicide. It seemed that enhanced tolerance to $\mathrm{H}_{2} \mathrm{O}_{2}$ was not a common mechanism for hormetic effects of a fungicide on virulence of plant fungal pathogens.

Flusilazole in PDA at concentrations of 0.005 to $0.16 \mu \mathrm{g} / \mathrm{ml}$ inhibited mycelial growth of S. sclerotiorum. However, after the mycelia were inoculated on rapeseed leaves, the inhibited mycelia exhibited higher virulence than the fungicide-free control. For isolate GS-7, after mycelia inhibited by $48.4 \%$ in terms of colony radial growth on PDA were inoculated on rapeseed plants, virulence stimulation was significantly higher than the control. However, after mycelia inhibited by $59.7 \%$ were inoculated on plants, virulence was slightly lower than the control. For isolate HN-24, mycelia inhibited by $14.4 \%$ showed significantly greater virulence, whereas mycelia inhibited by $24.9 \%$ showed slightly lower virulence than the control. These results indicate that the relationship between mycelia inhibition and subsequent virulence stimulation may vary considerably even among different isolates of the same pathogen species. In the field, a portion of mycelia or spores will survive fungicide applications. Further investigations are warranted to determine whether and to what extent the inhibited mycelia and surviving spores will have stimulated virulence to plants in the field.

\section{Acknowledgments}

This study was supported by the National Natural Science Foundation of China (31371964).

\section{Literature Cited}

Adams, P. B., and Ayres, B. E. 1979. Ecology of Sclerotinia species. Phytopathology 69:896-899.

Baraldi, E., Mari, M., Chierici, E., Pondrelli, M., Bertolini, P., and Pratella, G. C. 2003. Studies on thiabendazole resistance of Penicillium expansum of pears: Pathogenic fitness and genetic characterization. Plant Pathol. 52:362-370.

Becher, R., and Wirsel, S. G. 2012. Fungal cytochrome P450 sterol 14 $\alpha$-demethylase (CYP51) and azole resistance in plant and human pathogens. Appl. Microbiol. Biotechnol. 95:825-840.

Boland, G. J., and Hall, R. 1994. Index of plant hosts of Sclerotinia sclerotiorum. Can. J. Plant Pathol. 16:93-108.

Bolton, M. C., Thomma, B. P., and Nelson, B. D. 2006. Sclerotinia sclerotiorum (Lib.) de Bary: Biology and molecular traits of a cosmopolitan pathogen. Mol. Plant Pathol. 7:1-16.

Calabrese, E. J. 2001. Overcompensation stimulation: A mechanism for hormetic effects. Crit. Rev. Toxicol. 31:425-470.

Calabrese, E. J. 2013. Hormetic mechanisms. Crit. Rev. Toxicol. 43:580-606.

Calabrese, E. J. 2015a. Hormesis: Principles and applications. Homeopathy 104: 69-82.

Calabrese, E. J. 2015b. Hormesis within a mechanistic context. Homeopathy 104 90-96.

Calabrese, E. J. 2016a. Preconditioning is hormesis part I: Documentation, doseresponse features and mechanistic foundations. Pharmacol. Res. 110:242-264.

Calabrese, E. J. 2016b. Preconditioning is hormesis part II: How the conditioning dose mediates protection: Dose optimization within temporal and mechanistic frameworks. Pharmacol. Res. 110:265-275.

Calabrese, E. J., and Blain, R. B. 2011. The hormesis database: The occurrence of hormetic dose responses in the toxicological literature. Regul. Toxicol Pharmacol. 61:73-81.

Di, Y. L., Cong, M. L., Zhang, R., and Zhu, F. X. 2016a. Hormetic effects of trifloxystrobin on aggressiveness of Sclerotinia sclerotiorum. Plant Dis. 100: 2113-2118.

Di, Y. L., Lu, X. M., Zhu, Z. Q., and Zhu, F. X. 2016b. Time-course of carbendazim stimulation on pathogenicity of Sclerotinia sclerotiorum indicates a direct stimulation mechanism. Plant Dis. 100:1454-1459.

Di, Y. L., Zhu, Z. Q., Lu, X. M., and Zhu, F. X. 2015. Pathogenicity stimulation of Sclerotinia sclerotiorum by subtoxic doses of carbendazim. Plant Dis. 99: $1342-1346$.

Firoz, M. J., Xiao, X., Zhu, F. X., Fu, Y. P., Jiang, D. H., Schnabel, G., and Luo, C. X. 2016. Exploring mechanisms of resistance to dimethachlone in Sclerotinia sclerotiorum. Pest Manage. Sci. 72:770-779.

Flores, F. J., and Garzón, C. D. 2013. Detection and assessment of chemical hormesis on the radial growth in vitro of oomycetes and fungal plant pathogens. Dose Response 11:361-373.

Garzón, C. D., Molineros, J. E., Yanez, J. M., Flores, F. J., Jimenez-Gasco, M. M. and Moorman, G. W. 2011. Sublethal doses of mefenoxam enhance Pythium damping-off of geranium. Plant Dis. 95:1233-1238.

Lu, X. M., Zhu, Z. Q., Di, Y. L., and Zhu, F. X. 2015. Baseline sensitivity and toxic action of flusilazole to Sclerotinia sclerotiorum. Crop Prot. 78:92-98.

Moorman, G. W., and Kim, S. H. 2004. Species of Pythium from greenhouses in Pennsylvania exhibit resistance to propamocarb and mefenoxam. Plant Dis. 88: 630-632.

USDA. 2012. National strategic plan for the Sclerotinia research initiative-Integrated research for disease management in sunflower, canola, dry bean, pea \& lentils and soybean. Online publication. United States Department of Agriculture. https:// www.ars.usda.gov/Research/docs.htm?docid=20122

Wang, Y., Hou, Y. P., Chen, C. J., and Zhou, M. G. 2014. Detection of resistance in Sclerotinia sclerotiorum to carbendazim and dimethachlon in Jiangsu Province of China. Australas. Plant Pathol. 43:307-312.

Williams, B., Kabbage, M., Kim, H. J., Britt, R., and Dickman, M. B. 2011 Tipping the balance: Sclerotinia sclerotiorum secreted oxalic acid suppresses host defenses by manipulating the host redox environment. PLoS Pathog 7: e1002107.

Wojtaszek, P. 1997. Oxidative burst: An early plant response to pathogen infection. Biochem. J. 322:681-692.

Zhang, S., Panaccione, S. G., and Gallegly, M. E. 1997. Metalaxyl stimulation of growth of isolates of Phytophthora infestans. Mycologia 89:289-292.

Zhou, F., Liang, H. J., Di, Y. L., You, H., and Zhu, F. X. 2014. Stimulatory effects of sublethal doses of dimethachlon on Sclerotinia sclerotiorum. Plant Dis. 98: 1364-1370 